\title{
Relativistic Fe line emission and highly photoionized absorption in GRO J1655-40
}

\author{
M. Díaz Trigo ${ }^{1, \star}$, A. Parmar ${ }^{2}$, J.M. Miller ${ }^{3}$, and E. Kuulkers ${ }^{1}$ \\ 1 Science Operations and Data Systems Division, Research and Scientific Support Department of ESA, ESAC, Apartado \\ 50727, 28080 Madrid, Spain \\ 2 Astrophysics Mission Division, Research and Scientific Support Department of ESA, ESTEC, Postbus 299, NL-2200 \\ AG Noordwijk, The Netherlands \\ 3 Department of Astronomy, University of Michigan, 500 Church Street, Dennison 814, Ann Arbor, MI 48109, USA
}

Received 2006 Sep 1, accepted 2006 Oct 4

Published online 2006 Nov 28

Key words X-rays: binaries - Accretion, accretion disks - X-rays: individual: GRO J1655-40

We report on two XMM-Newton and simultaneous INTEGRAL observations of the microquasar GRO J1655-40 during its 2005 outburst. The source was most probably in its high-soft state during both observations. There is evidence for the presence of both a relativistically broadened Fe line providing strong support for the existence of a spinning black hole, and a highly photo-ionized absorber. The photo-ionized absorber is responsible for strong $\mathrm{K}$ absorption lines of Fe XXV and Fe XXVI in the EPIC pn spectra. The parameters of the highly-ionized absorber were different during the two observations. A less ionized absorber is present in the second observation, where the $0.5-200 \mathrm{keV}$ luminosity of GRO $\mathrm{J} 1655-40$ decreased by around a half.

\section{Introduction}

The X-ray nova GRO J1655-40 was discovered using the Burst and Transient Source Experiment (BATSE) onboard the Compton Gamma Ray Observatory (Zhang et al. 1994). Soon after the discovery radio observations revealed apparently superluminal relativistic jets moving in opposite directions almost perpendicular to the line of sight $\left(85^{\circ}\right)$ with a velocity of 0.92 c (Hjellming \& Rupen 1995; Tingay et al. 1995). Optical observations, obtained when the system had returned to quiescence, showed that the companion star is a F3-5 giant or sub-giant in a 2.62 day orbit around a 5$8 \mathrm{M}_{\odot}$ compact object, which is almost certainly a black hole (Orosz \& Bailyn 1997). GRO J1655-40 has been observed to undergo deep absorption dips (Kuulkers et al. 1998) consistent with the source being observed at an inclination angle of $\sim 70^{\circ}$ (Van der Hooft et al. 1998).

GRO J1655-40 has been subject of a considerable number of X-ray timing studies, presenting the highestfrequency quasi-periodic oscillations (QPOs) yet seen in a black hole (450 Hz, Strohmayer 2001).

Narrow X-ray absorption lines from highly ionized Fe were first detected from GRO J1655-40 (Ueda et al. 1998) and from another superluminal jet source GRS 1915+105 (Kotani et al. 2000). Similar features were subsequently detected from the low-mass X-ray binary (LMXB) GX 13+1 (Ueda et al. 2001) and from all the bright dipping LMXBs observed with XMM-Newton (Díaz Trigo et al. 2006a). The

\footnotetext{
^ Corresponding author: Maria.Diaz@sciops.esa.int
}

changes in the X-ray continuum and the Fe absorption features during dips are explained as resulting primarily from an increase in column density and a decrease in the ionization state of a highly-ionized absorber (Boirin et al. 2005; Díaz Trigo et al. 2006a). Since dipping sources are simply normal LMXBs viewed from close to the orbital plane, this implies that ionized absorbers are a common feature of LMXBs. Outside of the dips, the properties of the absorption features do not vary strongly with orbital phase. This suggests that the ionized plasma in LMXBs has a cylindrical geometry with a maximum column density close to the plane of the accretion disk.

Recently Chandra HETGS observations of the black hole candidates GX 339-4, XTEJ1650-500 and H 1743-322 (Miller et al. 2004, 2006) have revealed the presence of variable, blue-shifted, highly-ionized absorption features which are interpreted as evidence for outflows. While Fe XXV and Fe XXVI features are present in the H 1743-322 spectrum, GX 339-4 and XTEJ1650-500 show O VIII and Ne IX or Ne II features from less ionized material. These features suggest that a warm absorber analogous to those seen in many Seyfert galaxies is present in systems such as GX339-4 and XTEJ1650-500. In contrast, the absorber present in $\mathrm{H} 1743-322$ is more highly ionized.

We report on two simultaneous XMM-Newton and INTEGRAL observations of GRO J1655-40 obtained during a recent outburst that started around 2005 February 17 and reached a maximum intensity of $\sim 5 \mathrm{Crab}$ during flares around 2005 May 20. RXTE monitored the outburst and 
near-simultaneous observations to the XMM-Newton and INTEGRAL observations are available. Figure 1 shows the RXTE All-Sky Monitor (ASM) lightcurve of part of the 2005 outburst with the times of the XMM-Newton and INTEGRAL observations indicated.

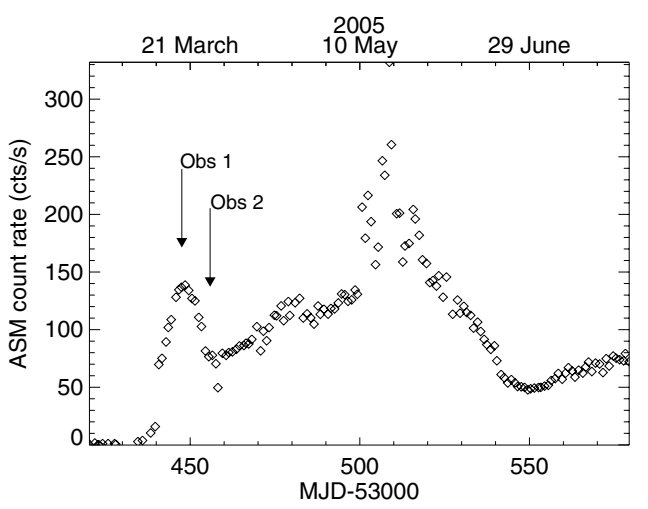

Fig. 1 ASM 1.5-12 keV lightcurve of GROJ1655-40. The times of the XMM-Newton and INTEGRAL observations reported here are indicated with arrows.

\section{Observations, data analysis and results}

We analysed two XMM-Newton observations of GRO J1655-40 taken on 2005 March 18 between 15:47 and 22:25 UTC for 23.8 ks (hereafter called Obs 1) and on 2005 March 27 between 08:43 and 14:56 UTC for 22.4 ks (hereafter called Obs 2) and the two simultaneous INTEGRAL observations performed between 2005 March 18 07:42 and 2005 March 19 04:22 UTC for $70 \mathrm{ks}$ and between 2005 March 26 19:00 and 2005 March 28 00:56 UTC for $100 \mathrm{ks}$. The EPIC pn camera was operated in burst mode due to the high count rate of the source $\left(\gtrsim 5000 \mathrm{~s}^{-1}\right)$. For the same reason the EPIC MOS cameras were not operated. RGS2 was operated in its normal spectroscopy mode with all CCD chips read in parallel. However, the individual RGS1 CCD chips were read out sequentially to reduce pile-up.

We first fit the $0.5-10 \mathrm{keV}$ EPIC pn spectra together with the 5-20 keV JEM-X and the 20-200 keV ISGRI spectra with a model consisting of a disk-blackbody and a power law, both modified by photo-electric absorption from neutral material (model tbabs* $(\mathrm{db} b+p o)$ in XSPEC). The fits are unacceptable with $\chi_{\nu}^{2}$ of 9.3 and 19.0 for 293 and 256 degrees of freedom (d.o.f.), for Obs 1 and Obs 2, respectively. The poor fit quality is mainly due to the presence of strong absorption features near $7 \mathrm{keV}$ and strong emission features in the pn below $3 \mathrm{keV}$. A broad emission feature is superposed on the $7 \mathrm{keV}$ absorption features. Structured residuals near $2.3 \mathrm{keV}$ are probably due to an incorrect instrumental modeling of the Au mirror edges, whilst those near $1.8 \mathrm{keV}$ are probably due to an incorrect modeling of the $\mathrm{Si}$ absorption in the CCD detectors. In addition, at the
Table 1 Best-fits to the $0.5-10 \mathrm{keV}$ EPIC pn, 5-20 $\mathrm{keV}$ JEM-X and 20-200 keV ISGRI spectra using the tbabs *warmabs * (di skbb+po) + tbabs * ( laor $2+$ gau $1+$ gau $\left._{2}+\operatorname{gau}_{3}+\mathrm{gau}_{4}\right)+\mathrm{gau}_{5}$ model $. k_{\mathrm{pl}}, k_{\mathrm{bb}}$ and $k_{\text {gau }}$ are the normalizations of the power law, disk-blackbody and emission Gaussian lines, respectively. $\mathrm{L}_{0.5-200 \mathrm{keV}}$ is the luminosity between 0.5 and $200 \mathrm{keV}$ and has been calculated assuming a distance of $3.2 \mathrm{kpc}$.

\begin{tabular}{|c|c|c|}
\hline & Obs 1 & Obs 2 \\
\hline $\begin{array}{lr}\Gamma & \text { po } \\
k_{\mathrm{pl}} & \\
\left(\mathrm{ph} \mathrm{keV} \mathrm{km}^{-1} \mathrm{~s}^{-1} \text { at } 1 \mathrm{keV}\right)\end{array}$ & $\begin{array}{l}2.23 \pm 0.02 \\
0.69 \pm 0.04\end{array}$ & $\begin{array}{c}3.14 \pm 0.02 \\
4.5 \pm 0.2\end{array}$ \\
\hline $\begin{array}{ll}k T_{\mathrm{bb}}(\mathrm{keV}) & \text { diskbb } \\
k_{\mathrm{bb}} & \\
\left(\left(R_{\mathrm{in}}(\mathrm{km}) /\left(D_{10}(\mathrm{kpc})\right)\right)^{2} * \cos \theta\right)\end{array}$ & $\begin{array}{c}1.320 \pm 0.003 \\
849 \pm 5\end{array}$ & $\begin{array}{c}1.303 \pm 0.003 \\
434{ }_{-3}^{+6}\end{array}$ \\
\hline$N_{\mathrm{H}}^{\mathrm{abs}}\left(10^{22} \mathrm{~cm}^{-2}\right) \quad$ abs & $0.665_{-0.003}^{+0.002}$ & $0.77_{-0.01}^{+0.02}$ \\
\hline $\begin{array}{l}N_{\mathrm{H}}^{\text {warmabs }}\left(10^{22} \mathrm{~cm}^{-2}\right) \\
\log (\xi)\left(\mathrm{erg} \mathrm{cm} \mathrm{s}^{-1}\right) \\
\sigma_{\mathrm{v}}\left(\mathrm{km} \mathrm{s}^{-1}\right)\end{array}$ & $\begin{aligned} 5.2 & \pm 1.0 \\
3.60 & \pm 0.04 \\
3500 & \pm 900\end{aligned}$ & $\begin{array}{c}1.5 \pm 1.2 \\
3.30 \pm 0.04 \\
5900 \pm 1200\end{array}$ \\
\hline $\begin{array}{l}E_{\text {gau }}(\mathrm{keV}) \\
k_{\text {gau }}\left(\mathrm{ph} \mathrm{cm}^{-2} \mathrm{~s}^{-1}\right) \\
q_{1} \\
r_{\text {in }}\left(\mathrm{GM} / \mathrm{c}^{2}\right) \\
i \text { (deg.) } \\
q_{2} \\
r_{\text {break }}\left(\mathrm{GM} / \mathrm{c}^{2}\right)\end{array}$ & $\begin{aligned} & 6.60 \pm 0.06 \\
& 0.25 \pm 0.02 \\
& 8.9 \pm 0.2 \\
& 1.53 \pm 0.02 \\
& 52 \pm 1 \\
& 3.5_{-0.3}^{+0.5} \\
& 3.6 \pm 0.2\end{aligned}$ & $\begin{aligned} & 6.80_{-0.05}^{+0.03} \\
& 0.226 \pm 0.006 \\
&>9.9 \\
& 1.37 \pm 0.01 \\
& 63.2 \pm 0.2 \\
& 3.0 \pm 0.3 \\
& 3.10 \pm 0.09\end{aligned}$ \\
\hline $\begin{array}{l}E_{\text {gau }}(\mathrm{keV}) \\
\sigma(\mathrm{keV}) \\
k_{\text {gau }}\left(\mathrm{ph} \mathrm{cm}^{-2} \mathrm{~s}^{-1}\right)\end{array}$ & $\begin{array}{c}0.50(\mathrm{f}) \\
0(\mathrm{f}) \\
0.4 \pm 0.2\end{array}$ & $\begin{array}{c}0.50(\mathrm{f}) \\
0(\mathrm{f}) \\
2.0 \pm 0.3\end{array}$ \\
\hline $\begin{array}{l}E_{\text {gau }}(\mathrm{keV}) \\
\sigma(\mathrm{keV}) \\
k_{\text {gau }}\left(\mathrm{ph} \mathrm{cm}^{-2} \mathrm{~s}^{-1}\right)\end{array}$ & $\begin{array}{c}0.60(\mathrm{f}) \\
<0.02 \\
0.3 \pm 0.1\end{array}$ & $\begin{array}{l}0.60(\text { f) } \\
<0.04 \\
<0.4\end{array}$ \\
\hline $\begin{array}{l}E_{\text {gau }}(\mathrm{keV}) \\
\sigma(\mathrm{keV}) \\
k_{\text {gau }}\left(\mathrm{ph} \mathrm{cm}^{-2} \mathrm{~s}^{-1}\right)\end{array}$ & $\begin{array}{l}0.922(f) \\
>0.1 \\
<0.03\end{array}$ & $\begin{array}{c}0.922(\mathrm{f}) \\
>0.1 \\
0.25_{-0.05}^{+0.03}\end{array}$ \\
\hline $\begin{array}{l}E_{\text {gau }}(\mathrm{keV}) \\
\sigma(\mathrm{keV}) \\
k_{\text {gau }}\left(\mathrm{ph} \mathrm{cm}^{-2} \mathrm{~s}^{-1}\right)\end{array}$ & $\begin{array}{c}1.022(f) \\
0.01_{-0.01}^{+0.09} \\
0.11 \pm 0.03\end{array}$ & $\begin{array}{c}1.022(\mathrm{f}) \\
>0.1 \\
0.27 \pm 0.03\end{array}$ \\
\hline $\begin{array}{l}E_{\text {gau }}(\mathrm{keV}) \\
\sigma(\mathrm{keV}) \\
k_{\text {gau }}\left(\mathrm{ph} \mathrm{cm}^{-2} \mathrm{~s}^{-1}\right)\end{array}$ & $\begin{array}{c}2.28 \pm 0.02 \\
0 \text { (f) } \\
0.039 \pm 0.008\end{array}$ & $\begin{array}{c}2.29 \pm 0.03 \\
0(f) \\
0.008 \pm 0.004\end{array}$ \\
\hline $\begin{array}{l}\chi_{\nu}^{2} \\
\text { d.o.f. }\end{array}$ & $\begin{array}{l}1.27 \\
274\end{array}$ & $\begin{array}{l}1.85 \\
237\end{array}$ \\
\hline$L_{0.5-200 \mathrm{keV}}\left(10^{37} \mathrm{erg} \mathrm{s}^{-1}\right)$ & 7.68 & 3.95 \\
\hline
\end{tabular}

energies of these edges there is a sharp drop in the effective area of the pn. Both effects do not appear to be well accounted for in the EPIC pn calibration. An excess in the pn spectra is detected at $\sim 0.6 \mathrm{keV}$ and can be modeled by a Gaussian emission line. This feature is present in both observations and could be emission from O VII. In addition, an absorption feature in the pn spectra is detected at $\sim 0.5 \mathrm{keV}$. Several absorption features are detected in the RGS at this 

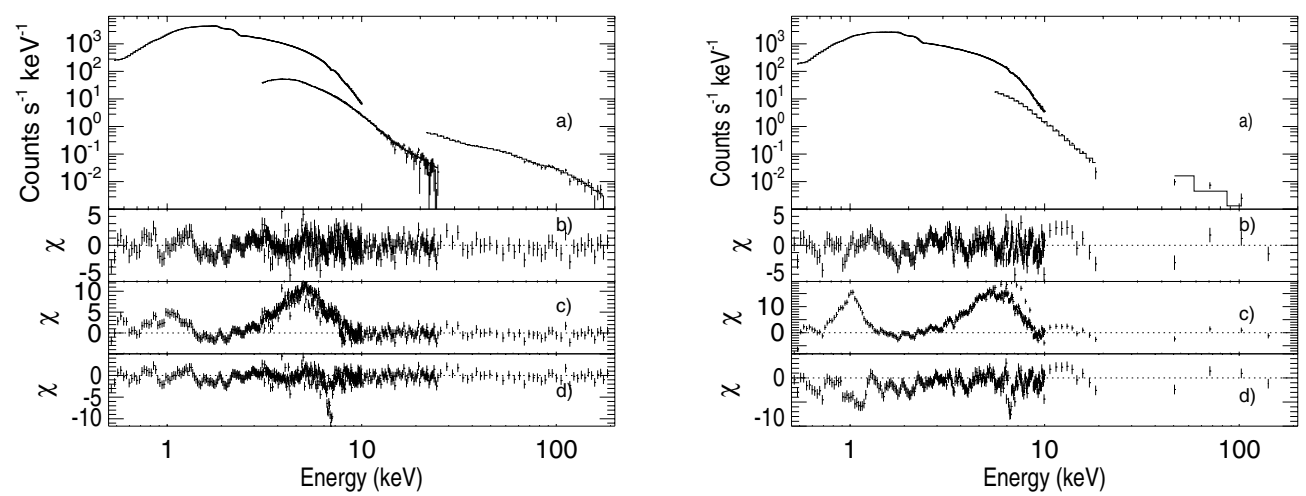

Fig. 2 (a) GRO J1655-40 EPIC pn, JEM-X and ISGRI spectra from Obs 1 (left) and Obs 2 (right) fit with a model consisting of diskblackbody (dbb) and power-law (po) components modified by absorption from neutral (tbabs) and ionized (warmabs) material together with a relativistic emission line (laor2), four emission Gaussians $\left(\mathrm{gau}_{1-4}\right)$ modified by absorption from neutral material (tbabs) and a Gaussian $\left(\mathrm{gau}_{5}\right.$ ) to account for calibration uncertainties. (b) Residuals in units of standard deviation from the above model. (c) Residuals when the normalisations of the laor 2 component, and the Gaussians are set to 0 . The laor 2 component indicates relativistic broadening. (d) Residuals when $N_{\mathrm{H}}^{\text {warmabs }}$ is set to 0 . Absorption lines are clearly visible near $7 \mathrm{keV}$. The best-fit parameters are given in Table 1 .

energy and are attributed to O I and O II (Díaz Trigo et al. 2006b). Finally, an excess near $1 \mathrm{keV}$ is detected in the EPIC pn spectrum. This feature is detected in a number of $\mathrm{X}$-ray binaries and has been previously modeled either as an emission line, or as an edge, and its nature is unclear. If the feature has an instrumental origin it might be expected to be stronger in the most luminous observation. The fact that the feature is very strong in Obs 2 and only a weak excess is observed in Obs 1 could indicate that it has an astrophysical origin and its energy is consistent with a blend of Ne IX and Ne X emission, or Fe L emission.

We included a narrow Gaussian emission feature at $2.3 \mathrm{keV}\left(\mathrm{gau}_{5}\right)$ to account for the burst mode calibration deficiencies discussed previously. A Gaussian absorption line gau $_{1}$ is added to account for the absorption at $\sim 0.5 \mathrm{keV}$. The excesses at $\sim 0.6 \mathrm{keV}$ and $\sim 1 \mathrm{keV}$ can be modeled by three Gaussian emission lines, gau $2-4$. The first Gaussian accounts for the excess at $0.6 \mathrm{keV}$. The other two Gaussians account for the excess at $1 \mathrm{keV}$ and their energies are fixed at 0.922 and $1.022 \mathrm{keV}$, the energies of Ne IX and $\mathrm{Ne} X$. This reduces the $\chi_{\nu}^{2}$ to 8.9 for 284 d.o.f for Obs 1 and to 8.7 for 247 d.o.f. for Obs 2.

To account for the complex residuals near $7 \mathrm{keV}$, absorption from a photo-ionized plasma (warmabs model within XSPEC) and a relativistic emission line (laor 2 ) modified by neutral absorption were added to the model. The laor 2 model describes the line profile expected when an accretion disk orbiting a black hole with maximum angular momentum is irradiated by a source of hard X-rays. The emissivity profile assumed is a broken power-law. The highly-ionized absorber models four absorption features, which are ficantly detected in both observations and consistent with absorption from Fe XXV and Fe XXVI Ly $\alpha$ and Ly $\beta$. During Obs 1, the $E W$ of the Fe XXV Ly $\alpha$ absorption feature is smaller than that of the Fe XXVI Ly $\alpha$, whilst the opposite occurs for Obs 2, indicating a less ionized absorber. The $\chi_{\nu}^{2}$ of the fit is significantly reduced to 1.27 for 274 d.o.f. and 1.85 for 237 d.o.f. for Obs 1 and Obs 2, respectively. The parameters of the best-fit model are given in Table 1. Figure 2 shows the best-fit model and residuals.

We note that during Obs 2, absorption features of $\mathrm{Ne} X$ and Fe XXIV with a blue-shift of $-540 \pm 120 \mathrm{~km} \mathrm{~s}^{-1}$ are detected in the 0.3-1.9 keV RGS spectrum (Díaz Trigo et al. 2006b), in addition to the EPIC pn Fe XXV and Fe XXVI features. A range of absorbers with different temperature or density is not required to explain the features at $\sim 7 \mathrm{keV}$ and below $2 \mathrm{keV}$.

We do not observe a strong variability during Obs 2, where the EPIC pn lightcurve shows a count rate of $\sim 160$ $\mathrm{s}^{-1}$ and a nearly constant hardness ratio. In Obs 1 , the pn lightcurve shows an increase in the count rate from $\sim 260$ to $\sim 300 \mathrm{~s}^{-1}$ which is associated with an increase of the hardness ratio. However, when we divide Obs 1 in two intervals and fit the corresponding EPIC pn spectra separately, the parameters of the fit are consistent within the errors, except for the index of the power-law component, which is slightly lower in the second interval.

\section{Discussion}

We have analysed two observations of GRO J1655-40 during its 2005 outburst. The source is clearly in the highsoft state, showing an X-ray emission dominated by thermal emission, which is $\gtrsim 90 \%$ the $2-10 \mathrm{keV}$ luminosity, probably from the optically thick accretion disk, and a weak, steep hard X-ray power law. The continuum is well described by a disk-blackbody with a temperature of $\sim 1.3 \mathrm{keV}$ and a power-law component with a photon index of $\sim 2.2-3.1$.

A highly-ionized absorber is observed superposed on the continuum in both observations. The absorber is less ionized and has a smaller column density during Obs 2. Presumably, the ionized absorber is in outflow in both observations, but we can only give upper limits (the EPIC pn energy resolution is $\sim 1000 \mathrm{~km} \mathrm{~s}^{-1}$ at $6 \mathrm{keV}$ ) to the outflow velocity 
in Obs 1 due to the absence of features in the RGS spectrum. The value of the ionization parameter of the absorber is directly related to the luminosity $\left(\xi=L / n_{\mathrm{e}} r^{2}\right)$. Therefore a lower degree of ionization is expected in Obs 2 , which has a lower luminosity, as detected.

Narrow X-ray absorption lines from highly ionized $\mathrm{Fe}$ were first detected in superluminal jet sources and subsequently in an increasing number of low-mass X-ray binaries observed by XMM-Newton. Recently, Chandra HETGS observations of a number of black hole candidates have revealed the presence of variable, blue-shifted, highly-ionized absorption features which are interpreted as evidence for outflows. The detection of an outflowing highly-ionized absorber in GROJ1655-40 confirms that the outflowing winds present in AGNs are also a common feature of microquasars. In contrast, no blueshifts have been detected in any of the highly-ionized absorbers present in dipping LMXBs. However, these results are all obtained with the EPIC pn, which has a factor $\sim 4$ poorer resolution than the HETGS at $6 \mathrm{keV}$ limiting the sensitivity to shifts $\gtrsim 1000 \mathrm{~km} \mathrm{~s}^{-1}$.

The detection of a different column density and ionization state of the absorber in different phases of the outburst establishes a clear link between the variability of the warm absorber and the central accretion engine. The long dynamical time scales make it difficult to perform similar studies in AGNs. Thus future observations of galactic black holes may also provide insights in accretion processes in AGNs.

A relativistically broadened $\mathrm{Fe} \mathrm{K} \alpha$ emission line is detected in both observations. The inner emission radius of the line is smaller than $6 R_{\mathrm{g}}$, indicating that GRO J1655-40 harbors a black hole with significant spin. A further indication of the existence of a spinning black hole in GROJ1655-40 is the presence of high-frequency QPOs. All the parameters of the line, except the emissivity, are consistent with the relativistically broadened $\mathrm{Fe} \mathrm{K} \alpha$ line previously detected by ASCA (Miller et al. 2005). The energy of the lines, 6.60 and $6.80 \mathrm{keV}$, is consistent with ionized emission from Fe XXV and lower ionization stages from $\mathrm{Fe}$, like Fe XXIV and Fe XXIII for Obs 1, and from Fe XXV and $\mathrm{Fe} X X V I$ for Obs 2 . Ionized $\mathrm{Fe} \mathrm{K}$ emission is expected from a disk with such a high temperature $(k T \sim 1.3 \mathrm{keV})$. The discrepancy in the emissivity, $\sim 9-10$ in our observations compared to $\sim 5.5$ in ASCA observations, may be a consequence of the different model chosen to perform the fits. The spectra fit in this paper show highly ionized absorption lines, which are modeled with a photo-ionized absorber. The absorption is strong and causes a change in the continuum. The emission line occurs at the same energy interval than the absorption lines and edges, and therefore an absent or deficient modeling of the absorption (e.g. by including the lines and edges independently) will vary the parameters of the emission feature. Further, the ASCA fits include a smeared edge at $8 \mathrm{keV}$ to simulate reflection from the disk, which is not included in our fits. Finally, the inclination in the ASCA fits $\left(45_{-5}^{+15}\right.$ degrees), though consistent within the errors, is lower than the one in our fits $(52 \pm$
1 and $63.2 \pm 0.2$ degrees). This may explain partially the high emissivity of our fits, since an increase in the emissivity produces the same effect as a lower inclination in the relativistic line. The simultaneous modeling of the absorption features and of the relativistic $\mathrm{Fe} \mathrm{K}$ emission line is crucial in order to obtain realistic values for the parameters of the model. It has been argued (e.g., Done \& Gierlinski 2006) that relativistic smearing can be significantly reduced if there is also $\mathrm{Fe} \mathrm{K}$ line absorption from an outflowing disk wind. We find that both an extremely relativistic emission line, confirming the existence of a spinning black hole, and strong ionized absorption are present in the outburst spectra of GRO J1655-40.

A narrow emission line is observed at $0.6 \mathrm{keV}$ in $\mathrm{Obs} 1$, which could be associated to emission from O VII, if its astrophysical origin is confirmed. However, its non-detection in the RGS data may indicate that the line has an instrumental origin. The feature at $1 \mathrm{keV}$ in Obs 2 has been observed in other sources and its origin is unclear. The fact that it is only detected in the less luminous GRO J1655-40 observation indicates that it could be of astrophysical origin. In that case, it could be related to a combination of Ne IX and $\mathrm{Ne} X$ or the Fe L complex.

Acknowledgements. Based on observations obtained with XMMNewton, an ESA science mission with instruments and contributions directly funded by ESA member states and the USA (NASA), and on observations with INTEGRAL, an ESA project with instruments and science data centre funded by ESA member states (especially the PI countries: Denmark, France, Germany, Italy, Switzerland and Spain), Czech Republic and Poland, and with the participation of Russia and USA. M. Díaz Trigo acknowledges an ESA Research Fellowship. We thank the RXTE instrument teams at MIT and NASA/GSFC for providing the ASM lightcurve.

\section{References}

Boirin, L., Méndez, M., Díaz Trigo, M., Parmar, A., Kaastra, J.: 2005, A\&A 436, 195

Díaz Trigo, M., Parmar, A., Boirin, L., Méndez, M., Kaastra, J.: 2006a, A\&A 445, 179

Díaz Trigo, M., Parmar, A., Miller, J., Kuulkers, E.: 2006b, A\&A submitted

Hjellming, R.M., Rupen, M.P.: 1995, Nature 375, 464

Kotani, T., Ebisawa, K., Dotani, T., et al.: 2000, ApJ 539, 413

Kuulkers, E., Wijnands, R., Belloni, T., et al.: 1998, ApJ 494, 753

Miller, J.M., Raymond, J., Fabian, A.C., et al.: 2004, ApJ 601, 450

Miller, J.M., Fabian, A.C., Nowak, M.A., Lewin, W.H.G.: 2005, in: M. Novello, S. Perez Bergliaffa, R. Ruffini (eds.), The Tenth M. Grossman Meeting, p. 1296

Miller, J.M., Raymond, J., Homan, J., et al.: 2006, ApJ, in press

Orosz, J.A., Baylin, C.D.: 1997, ApJ 477, 876

Ueda, Y., Inoue, H., Tanaka, Y., et al.: 1998, ApJ 492, 782

Ueda, Y., Asai, K., Yamaoka, K., Dotani, T., Inoue, H.: 2001, ApJ 556 L87

Van der Hooft, Heemskerk, M.H.M., Alberts, F., van Paradijs, J.: 1998, A\&A 329, 538

Tingay, S.J., Jauncey, D.L., Preston, R.A., et al.: 1995, Nature 374, 191

Zhang, S.N., Wilson, C.A., Harmon, B.A., et al.: 1994, IAU Circ. 6046,1 